\title{
Meta
}

Journal des traducteurs

Translators' Journal

\section{Le modèle d'analyse textuelle dialogique : la traduction poétique au-delà du contenu et de la forme}

\section{Aurélia Klimkiewicz}

Volume 45, numéro 2, juin 2000

URI : https://id.erudit.org/iderudit/003326ar

DOI : https://doi.org/10.7202/003326ar

Aller au sommaire du numéro

Éditeur(s)

Les Presses de l'Université de Montréal

ISSN

0026-0452 (imprimé)

1492-1421 (numérique)

Découvrir la revue

Citer cet article

Klimkiewicz, A. (2000). Le modèle d'analyse textuelle dialogique : la traduction poétique au-delà du contenu et de la forme. Meta, 45(2), 175-192.

https://doi.org/10.7202/003326ar

\section{Résumé de l'article}

Pour traduire un poème, comment résoudre le problème forme/contenu ? Rechercher la voix de l'auteur dans son émergence historique, aller à la rencontre de l'Autre pris dans sa finitude historique, faire ressortir le sens du dialogue dans sa pluralité discursive, ce sont des thèmes abordés dans cet article qui traite de la traduction poétique dialogique.Les remarques théoriques servent à commenter la traduction francaise de Chalamov" La chaussée ". 


\title{
Le modèle d'analyse textuelle dialogique: la traduction poétique au-delà du contenu et de la forme
}

\author{
AURÉLIA KLIMKIEWICZ \\ Université de Montréal, Montréal, Canada
}

\begin{abstract}
RÉSUMÉ
Pour traduire un poème, comment résoudre le problème forme/contenu ? Rechercher la voix de l'auteur dans son émergence historique, aller à la rencontre de l'Autre pris dans sa finitude historique, faire ressortir le sens du dialogue dans sa pluralité discursive, ce sont des thèmes abordés dans cet article qui traite de la traduction poétique dialogique. Les remarques théoriques servent à commenter la traduction francaise de Chalamov «La chaussée».
\end{abstract}

\section{ABSTRACT}

According to dialogical translation, to solve a problem of form and content, the translator should hear the author's voice in his/her historical context, should meet the Other confronted by finitude, should emphasize the discursive plurality of that dialogue. These theoretical presuppositions are applied to comment on French translation of Russian poet Shalamov.

\section{MOTS-CLÉS/KEYWORDS}

traduction poétique, dialogue, voix, compréhension, historicité

Le texte n'existe que dans le rapport établi avec ses lecteurs, ses interprètes et ses traducteurs. Plus l'ensemble de destinataires s'élargit, plus le texte se dévoile, s'enrichit ou s'actualise. Mais ce rapport, comment le définir? Quels éléments faut-il prendre en considération? Quels aspects méritent la priorité? Est-ce le contenu, la forme, l'intention de l'auteur, la subjectivité du lecteur, le contexte de la production?

Comme la vision du texte conditionne toute forme d'interprétation, ces questions deviennent pertinentes dans le domaine de la traduction si l'on admet que celle-ci est toujours motivée par un effort préalable de l'interprétation. Les distinctions tranchées au couteau entre auteur et traducteur, forme et contenu, style et sens, sourciers et ciblistes, fidélité et contamination, passivité et création, équivalence et non-équivalence deviennent moins radicales du moment où l'exercice de traduction est vu comme un processus dialogique, c'est-à-dire prenant place dans un événement qui est une rencontre avec l'autre, une relation basée sur une différence essentielle dans laquelle les sujets participant ne coïncideront jamais l'un avec l'autre.

Cet article soutient une réflexion portant tout d'abord sur la validité des distinctions dichotomiques qui continuent de façonner l'image de la traduction. Il s'agit particulièrement de l'opposition qui existe entre la forme et le contenu, opposition creusée, selon Meschonnic (1989), par les analyses structurales et linguistiques valorisant la langue comme système abstrait dont l'aspect physique et dynamique est réduit au statut résiduel de la fonction langagière.

Meta, $X L V, 2,2000$ 
Le dualisme du signe, qui sera notre point de départ, nous mènera à l'analyse dialogique qui dépasse autant les oppositions binaires que les synthèses axées sur la totalisation du savoir, et qui rejoint plutôt l'idée du métissage ${ }^{1}$ entre les langues, les cultures, les époques et les individus.

Si l'activité dialogique se trouve à la base de la traduction ou, inversement, si la traduction est a priori un processus dialogique, il faudrait reconnaitre l'importance de différents éléments qui rentrent en ligne de compte séparément, successivement ou simultanément. L'application de tel ou tel mode d'analyse ou d'orientation conceptuelle dépend du choix individuel du traducteur, du type de texte à analyser (on ne traduit pas de la même manière un pamphlet publicitaire, une description technique ou Dostoïevski) ou bien de paramètres socioéconomiques. Qui dit dialogue dit également multiplicité de voix et de lectures dont aucune n'est privilégiée. Le dernier mot n'appartient ni à l'auteur ni au traducteur. L'enjeu de traduction est beaucoup plus vaste: il englobe aussi le travail du correcteur, la politique d'une maison d'édition et l'horizon d'attente du public ciblé.

\section{L'EFFET STRUCTURALISTE DANS LA TRADUCTION POÉTIQUE}

$\mathrm{Au}$ tout début du $\mathrm{xx}^{\mathrm{e}}$ siècle, les formalistes russes prennent leurs distances avec l'historicisme qui prédomine dans les études littéraires et cherchent plus d'objectivité en élaborant des méthodes dites scientifiques, centrées sur la construction formelle du texte.

En Occident, la redécouverte du Cours de linguistique générale de F. de Saussure, publié initialement en 1916, révolutionne non seulement la linguistique mais connaît aussi un grand écho dans les milieux littéraires des années 1950. Inspirés par la notion de langue comme système, par la distinction entre la langue et la parole, entre le signifiant et le signifié, les structuralistes français, tout comme les formalistes russes, traiteront le texte comme un système, comme une construction formelle d'où seront exclus aussi bien la figure de l'auteur que l'objet, la réalité ou le référent extérieur au langage.

Le mal qui guette les traducteurs, selon Henri Meschonnic, est justement l'effet structuraliste inspiré par la notion saussurienne du signe ${ }^{2}$ ou plus précisément par les distinctions entre la parole et la langue, le signifiant et le signifié, le son et le sens, l'oralité et l'écriture. Ce dualisme, dont l'origine remonte à Platon, permet d'abandonner l'aspect discursif du langage, qui se manifeste dans sa corporalité et dans son historicité, pour se concentrer sur l'analyse de la langue-système où l'apport du sujet, par définition historique et auto-réfléchissant, est annihilé devant l'immanence de la langue qui parle toute seule et qui le transcende.

L'impact structuraliste, qui, selon Meschonnic, marque le domaine de la traduction poétique en France, s'explique par le fait que la poésie est traduite non pas à partir d'elle-même mais à travers le prisme d'une théorie du langage ${ }^{3}$. Dans ce cas, le sujet traduisant déshistorisé, au lieu de rejoindre la poésie dans son rythme vu comme «l'organisation continue du langage par un sujet» (Meschonnic 1989: 111), se sert d'un outil théorique qui organise le sens (Meschonnic 1989: 116). Cette démarche est justement celle qui passe sous silence les questions qu'un texte soulève, questions qui constituent la raison d'être de toute écriture: 
Dès qu'on lit sans question, on ne lit plus, on est, à l'inverse, dévoré par l' « objet» de la lecture. C'est bien alors qu'au lieu d'un sujet de la lecture, il n'y a plus que la créature d'un réseau grammatical, le réseau d'une logique plus puissante et qui a installé son monde. (Meschonnic 1989: 114)

Démontrer uniquement la production et les modes d'organisation du sens, d'où le sujet est d'emblée évacué et déresponsabilisé, est à l'opposé du rapport d'écoute avec le texte, écoute qui englobe l'intonation, le corps, la voix et la vie, tout «ce qui échappe au sens, c'est-à-dire au modèle du signe» (Meschonnic 1989: 203).

Tout comme l'imposture du signe, selon l'expression de Meschonnic, l'approche historisante (le texte comme produit d'une époque), psychologisante (la recherche de l'intention de l'auteur) ou sociologisante (le texte comme produit d'un milieu social) sont des prismes extérieurs qui s'imposent aux traducteurs et limitent ainsi le rapport direct entre un sujet traduisant et l'original. L'imposture des normes ou des théories élimine la conscience historique, sans laquelle le traducteur devient «mutilé, prisonnier de sa représentation du traduire et de celles qui véhiculent les discours sociaux du moment» (Berman 1995: 61).

\section{L'ANALYSE DIALOGIQUE}

L'approche dialogique du texte ne devient possible qu'après l'abandon du modèle classique de la connaissance, modèle monologique et descriptif, au profit du modèle dialogique, historique et centré sur la compréhension, qui, dès l'aube du $\mathrm{xx}^{\mathrm{e}}$ siècle, s'impose dans la culture occidentale.

Analogiquement, dans le domaine scientifique, ce changement correspond à l'abandon de la théorie newtonienne pour la relativité d'Einstein et la physique quantique. Dans le domaine philosophique, cette coupure épistémologique se traduit par le refus de la métaphysique axée sur le fondement unique, véridique et stable de toute forme de connaissance humaine. Il en va ainsi de l'histoire qui n'est plus vue comme un spectacle qui s'offre dans la continuité des événements chronologiques mais comme un site archéologique composé de morceaux éparpillés ici et là, dont la signification restera pour toujours présente/absente, dévoilée/cachée, éloquente/ muette. L'histoire ne se compose plus des faits qu'on décrit mais des événements qu'il faut comprendre et interpréter. L'Histoire devient l'histoire sans fin.

Mais c'est dans l'art où se sent le plus la coupure avec la tradition et le passé. L'art du $\mathrm{xx}^{\mathrm{e}}$ ne garantit plus rien et ne donne pas de réponses; il exprime plutôt le perpétuel effort d'exprimer l'ineffable et résiste même à l'esthétique si elle est comprise comme une réception immédiate de l'art par le biais du plaisir, de l'intuition et des sens où le récepteur s'abandonne à la création artistique par une sorte d'attirance dite naturelle, spontanée et innocente, c'est-à-dire sans comprendre ni même avoir besoin de comprendre le pourquoi de cette attirance ${ }^{4}$.

L'art contemporain se présente à nous dans son dépouillement, dans son austérité extrême. Il n'est ni l'objet d'admiration ni de consommation, mais il exprime le refus de la prison de l'esthétique, le refus de la représentation, du sens, de la vérité et de toute forme établie de création. L'art se retire de la vie et devient l'opacité, la nonreprésentation, le vide, l'indicible. Au lieu d'attirer le public, il crée une distance: là où le public réclame la beauté et l'harmonie, la facilité de réception et le plaisir, l'art offre l'éclatement du sens et la discontinuité, la distance et l'étrangeté, l'étonnement 
et la déstabilisation. Dans ces conditions, la lecture d'un texte, la contemplation d'un tableau ou d'une sculpture, l'écoute d'une pièce musicale exigent une réflexion basée sur un savoir préalable qui produira une compréhension, car nos sens ne répondent plus spontanément à cet univers opaque. Plus l'art s'éloigne de l'évidence de la réalité, plus il laisse de place à l'autre, à l'interprète, à son savoir et à sa créativité. Déstabilisé, le destinataire doit accomplir un travail d'interprète, travail qui le mène sur un chemin tortueux et incertain, travail qui le transforme en cours de route, qui le pousse aux limites sans toutefois mener à une destination précise et finale. Cette position éthique s'affirme par le fait qu'achever une interprétation veut dire ouvrir immédiatement la porte aux autres interprétations. Et par là continuer le dialogue.

Pour revenir à l'analyse dialogique du texte, elle correspond ainsi mieux aux exigences de l'art du $\mathrm{xx}^{\mathrm{e}}$ siècle. Dans ces conditions nouvelles, des questions fondamentales sont à poser conernant la traduction dont la théorie et la pédagogie semblent résister encore et contre tout au modèle de la connaissance contemporain que nous venons d'esquisser. Est-il possible de traduire un Beckett ou un Blanchot dans un esprit cartésien ${ }^{5}$ ? Ou à l'inverse, un Balzac ou un Tolstoï se traduiront-ils à partir de notre horizon épistémologique? Ou encore, face à l'art qui fait éclater le sens, que reste-t-il aux traducteurs: se conformer aux exigences de cet art en poussant encore plus loin le travail de la destruction (forme, sens, unité, etc.) ou bien y répondre par une réponse qui viserait plutôt l'affirmation créative ne serait-ce que fragmentaire? Et finalement, la neutralité du traducteur, du point de vue théorique, est-elle encore pensable?

L'analyse dialogique part du principe que tout texte est une entité hétérogène dépassant la distinction structuraliste entre la langue et la parole, entre la forme et le contenu. Tout texte prend sa naissance dans un dialogue qui émerge de la vie la plus concrète. Le but de l'écriture va au-delà du dévoilement d'une subjectivité pleine qui a quelque chose à dire, à communiquer, à faire passer auprès du public. Il dépasse aussi l'idée de l'écriture en tant que pur jeu de formes langagières qui se justifierait par le fait que tous les signes sont arbitraires (Saussure). L'écriture est, à son origine, un dialogue réel, dialogue simultané de l'auteur avec lui-même et avec les autres dans lequel se rencontrent le passé, le présent et même la projection vers l'avenir. De ce fait, l'écriture se définit comme dépassement de soi et ouverture vers l'autre.

Le dialogue qui nous concerne aujourd'hui ne s'identifie pas au dialogue de Platon : celui-ci sert à vaincre l'interlocuteur-adversaire, à le piéger en guettant une faille dans son raisonnement. Le véritable dialogue, comme l'a pensé Martin Buber, traducteur biblique du début du siècle, n'existe que dans l'immédiateté de la relation entre un Je et un $\mathrm{Tu}$, d'un être humain à un autre, relation régie par les sentiments, l'imprévu, la participation réciproque sans barrières ni limites. Ce moment privilégié est une rencontre réelle qui crée l'instant présent et «n'existe que s'il y a présence, rencontre, relation» (Buber 1969: 31).

Le domaine de la relation dégage une nouvelle épistémè, remplaçant celle du $\mathrm{XIX}^{\mathrm{e}}$ siècle, dominée par l'observation et la saisie de l'univers des objets par un sujet détaché. Le paradigme de l'autre qui surgit au même moment met fin à ce que Blanchot a appelé «la loi du jour» (1955: 323), c'est-à-dire au rêve métaphysique centré sur la lumière, la transparence de la pensée, l'affirmation, l'absolu. Dès lors, la connaissance se défait de la certitude et fait place au savoir transitoire et à la recherche inachevée. 
Parmi les penseurs du dialogue, le nom de Bakhtine mérite une attention particulière. Inspiré par Buber au moment où il travaillait sur Dostoïevski, il élabore sa théorie du dialogue qui aura un impact sur la pensée contemporaine dans de nombreux domaines: la philosophie, la critique littéraire, l'histoire, la sociologie, la linguistique, la culturologie, pour ne nommer que les plus importants.

Selon Bakhtine, le dialogue crée un univers inachevé, instable, chaotique et effrayant, tel que décrit dans les romans de Dostö̈evski. Le dialogue, cette activité humaine incessante et inépuisable, tout comme le Dire de Lévinas, «précède le langage» qui fait «découvrir le sujet comme le nœud d'une intrigue entre le Même et l'Autre» (Petrosino et Rolland 1984: 43) ayant lieu dans la discontinuité de la chaîne dialogique de la question et de la réponse. Le dialogue ou le Dire signifie le préoriginel, l'an-archique, d'avant l'archè, d'avant l'être (Petrosino et Rolland 1984: 45). Par contre, le Dit, c'est-à-dire le signe, le savoir, les théories, les systèmes et les représentations sont des formes cristallisées et imposées par une attitude monologique et non par l'inspiration divine, la rationalité, la logique ou autres. Bref, le mot adamique, pur et innocent, tout comme le stade pré-social et pré-linguistique, sont remplacés par l'activité dialogique incessante, multiple et créatrice; le mouvement linéaire et progressif de l'Histoire et du savoir se voit détrôné par les positions axiologiques les plus diverses qui sont seules garantes du dialogue; la vérité ne devient qu'une signification de nature événementielle. D'ailleurs, la physique quantique confirme que la réalité ne se présente pas comme un grand livre à déchiffrer (Galilée) ou comme une image fixe et statique (Newton) mais qu'elle se révèle en tant que processus infini et complexe d'actions et d'interactions.

Si l'existence n'est plus un spectacle, une vision ou un panoramique, selon le terme lévinasien (Lévinas 1974: 271) , où l'extériorité et l'intériorité ne font qu'un, mais une action, une conduite, un dialogue incessant et vivant, il va de soi que les notions métaphysiques de pensée, de connaissance et de création artistique subissent un changement radical et, par là, transforment les modes de leur mise en pratique.

Pour poursuivre notre réflexion sur l'analyse dialogique, il faut donc retenir le fait que le texte n'est plus un simple objet achevé qui se présente à la conscience unifiante de l'interprète grâce à sa logique sémantique ou bien grâce à ses structures immanentes. Le texte se dévoile plutôt dans son événementialité qui n'est ni récurrente ni observable mais renouvelable par des interprétations multiples.

Par sa définition, le dialogue a besoin de l'autre. Mais même le monologue, comme nous dit Bakhtine, est un dialogue avec les autres. L'être humain arrive dans un monde d'ores et déjà parlant, il arrive après coup. Il est l'être-jeté, selon Heidegger, à qui est donné la possibilité de choisir entre les deux modes d'être: le mode inauthentique où la tradition est acceptée telle quelle et le monde authentique qui se caractérise par le questionnement du passé dans lequel rien n'est définitif. Le mode d'être inauthentique serait proche de la notion bakhtinienne d'existence dans l'alibi, protégée par la couverture, les lois, des systèmes et des théories, n'exigeant aucune responsabilité. La présence de l'autre, la seule présence réelle et valide, a justement pour but d'ouvrir les entités et les systèmes qu'on croyait à tout jamais intacts et achevés. Cette présence devient obligatoire chaque fois que nous voulons questionner, comprendre ou communiquer quelque chose et non seulement constater, exprimer ou affirmer. Dès lors, notre propre connaissance s'ouvre, transcende ses limites, se transforme en accueillant les positions axiologiques des autres et en remettant en 
question les nôtres. Le dialogue, c'est-à-dire la rencontre avec l'autre qui est un $t u$, permet de sortir de ses propres limites ainsi que d'on, de la tradition, de la doxa, de l'intersubjectivité vue comme une pure transcendance qui régit la quotidienneté répétitive des individus dépourvus d'imagination créatrice.

Si l'on suit la pensée de Bakhtine, lire un texte est une rencontre avec l'autre. Peut-être est-il légitime de se mettre au niveau de la radicalité de la position lévinasienne et dire que le texte est un visage à part entière et la lecture un face-à-face avec son auteur. Cette rencontre se fait à travers le langage, sauf que celui-ci n'est plus un simple outil de communication ou une langue-système qui se parle toute seule, mais une manière de rejoindre l'autre, d'être en relation avec lui. Dans la perspective dialogique, le langage est une liberté, une créativité et une possibilité de dépasser son propre ego, sa propre tradition et temporalité. La lecture en tant qu'exercice esthétique basé sur le plaisir se transforme donc en une activité d'ordre éthique qui pose le comportement humain comme problématique et qui tente de redéfinir les principes qui régissent la vie commune.

Analyser, interpréter ou traduire un texte de manière dialogique, c'est entrer en dialogue avec l'auteur/narrateur et par lui avec les autres auteurs et individus qui lui servaient d'interlocuteurs. Selon Bakhtine, le texte est constitué de voix, ce qui n'est pas sans conséquence pour l'interprétation. Si l'unité d'analyse devient une voix et non seulement un discours, la lecture, quant à elle, devient une écoute. Et l'écoute, selon Bakhtine dans ses premiers écrits, se centre sur l'intonation qui exprime la position du sujet parlant face à l'objet de la discussion. L'écoute de l'intonation, de ce qui est individuel, non reproductible et événementiel, consiste à distinguer dans un mot, un énoncé ou un discours les tons émotionnels et volontaires. Le rapport direct entre le contenu sémantique du langage et la réalité, tel qu'envisagé et étudié en linguistique, se brise et se multiplie par le fait que ces tons expriment l'investissement personnel du sujet parlant, son opinion accord/désaccord, soumission/rejet, questionnement/ordre, etc. C'est pourquoi deux énoncés identiques du point de vue linguistique peuvent avoir deux significations différentes dans des situations variées. L'intonation est donc la signature de celui qui s'exprime ou produit un contenu, la preuve que celui-ci pense activement et qu'il est un être responsable. Nous sommes dans le domaine de l'acte, compris par Bakhtine comme un lien entre le fait et son sens, entre l'individuel et l'universel, entre la théorie et la vie, ou encore entre le contenu et l'intonation. La notion bakhtinienne de l'acte comporte des conséquences d'ordre éthique. D'un côté, comme nous l'avons déjà souligné, le langage n'est pas un pur outil de communication mais un moyen véritable et unique d'exprimer notre propre compréhension du monde et de l'existence, tout en affirmant leur portée limitée. D'un autre côté, si le contenu quelconque, par exemple d'un texte, d'une théorie, d'une loi, est plus qu'un Dit cristallisé une fois pour toutes, mais plutôt un Dire dans lequel retentissent encore des intonations, notre rapport à la culture, au savoir, à l'idéologie, à la religion doit subir une transformation radicale, car toute forme de connaissance prend ses sources dans l'événement ouvert qui est l'événement du dialogue.

Pour récapituler, il est important de retenir que l'analyse dialogique du texte devrait faire ressortir la multiplicité des voix qui y parlent. Â qui l'auteur s'adresse$\mathrm{t}$-il ? Quelles questions pose-t-il ? Est-il possible d'identifier des réponses qu'il donne à ceux qui l'ont précédé, à ceux qui lui sont contemporains et à ceux de la postério- 
rité? Soulève-t-il des problèmes nouveaux? Quelle est sa propre position? Répondre à ces questions est une tâche relativement facile s'il s'agit d'un texte contemporain. Tel n'est pas le cas quant aux textes provenant d'époques ou de cultures éloignées. Être à l'écoute des voix, savoir les reconnaître, signifie être à l'aise dans le contexte originaire de l'œuvre étudiée, d'où l'exigence de la lecture des textes périphériques avec lesquels l'auteur étudié pouvait entrer en contact. Une fois cette interprétation accomplie, l'analyse devrait consister à exprimer la compréhension du texte, à y laisser sa propre voix qui affirme les positions axiologiques du destinataire à partir de son excédentaire de la vision (ce que je perçois, l'autre ne le perçoit pas et inversement) qui est liée à sa place spatio-temporelle. Dans l'interprétation dialogique, le lecteur ne coïncide jamais avec l'auteur, mais il le croise sur le chemin de la réflexion personnelle. De plus, aucun d'eux ne détient le dernier mot. Les deux sont côte à côte, se côtoient, ou, comme dirait le Petit Prince, s'apprivoisent mutuellement.

\section{LE DIALOGUE ET LA TRADUCTION POÉTIQUE}

À titre d'exemple, nous avons choisi une traduction qui nous servira de prétexte à une brève réflexion sur les remarques théoriques exposées précédemment et à leur application.

Il s'agit du poème «La chaussée » écrit par le poète russe Varlam Chalamov en 1952, c'est-à-dire après avoir passé 17 ans en prison et en exil. Traduit en français par Christiane Loré, le poème est inclus dans le recueil Tout ou rien (1993: 156-157).

\section{CHOSSÉ}

Doroga tianetsia ot moria

Naverkh po beregou reki,

I gnout khrebty pod neiou gory,

Kak pod kanatom - bourlaki.

Oni prokhodiat droug za drougom V prozratchnykh severnykh notchakh. Oni oustali ot natougui, Ou nikh mozoli na pletchakh.

Oni tsepliaioutsia roukami

Za telegrafnye stolby

I vytiraiout oblakami

Svoi nakhmourennye lby.

Tcherez ovragui, tcherez iamy, Tcherez bolota i lessa Chagaiout gory vverkh i priamo I tachtchat more v nebessa.

\section{LA CHAUSSÉE}

La route s'étire de la mer Au-dessus de la rivière;

Aux monts elle fait ployer l'échine

Comme des haleurs sous leur cordage.

Sur la nuit transparente du Nord

Les monts se profilent tour à tour

Las de l'effort dont ils portent

Les stigmates sur l'épaule.

Cramponnés comme ils le peuvent

Aux poteaux télégraphiques,

Ils épongent sur les nuées

Leur front harassé.

Par-delà ravines et abîmes

Par-delà marais et forêts

S'élèvent droites les cimes

Tirant la mer jusqu'aux étoiles.

Nous proposons ici une traduction mot à mot de ce poème:

La route s'étire de la mer/Vers le haut au bord de la rivière,/Et les monts ploient leur échine sous son poids,/ Comme sous le cordage - les haleurs.

Ils se profilent un après l'autre/Dans les nuits nordiques transparentes./Ils se sont fatigués à cause de l'effort,/Ils ont leur peau durcie (les marques) aux épaules. 
Ils se cramponnent avec leurs mains/Aux poteaux télégraphiques/Et essuient avec les nuages/Leurs fronts assombris.

Par-delà ravines et cavités,/Par-delà marais et forêts/Marchent les montagnes vers le haut et tout droit/Et traînent la mer aux cieux.

Comparé à la traduction française, le contenu sémantique de l'original diffère sur deux plans. La traduction n'est pas toujours exacte au niveau terminologique et le niveau du langage est plus élevé dans la version française.

Voici des imprécisions terminologiques que nous avons remarquées:

- Le mouvement de la route qui s'étire vers le haut (ligne 2) est rendu par une image statique: "Au-dessus de la rivière».

- À la troisième ligne, l'agent de l'action est transféré des montagnes à la rivière, ce qui ne rend pas bien la pression réelle (la soumission sur le plan abstrait) que subissent les monts.

- L'expression «Dans (au milieu) des nuits transparentes» (1. 6 en russe) est traduite par «Sur la nuit transparente» qui évoque plutôt une image contemplative et détachée de l'action, d'autant que l'expression est employée au singulier (l. 5 en français).

- Le mot mozoli (1. 8) qui signifie les cors, le lieu où la peau est durcie à cause de l'effort physique soutenu, est surtraduit ou plutôt corrigé (les cors se forment sur les pieds et les mains et non pas sur les épaules) par «les stigmates» qui est un équivalent plus près de la réalité mais qui est imprégné de connotations religieuses. De plus, le pluriel «aux épaules» est rendu au singulier «sur l'épaule», comme si la traductrice établissait ici un lien avec la métaphore de la première strophe où des haleurs tirent des bateaux par un seul cordage.

- À la ligne 9, qui se lit en russe «Ils se cramponnent avec les mains », le mot «les mains» est absent dans la version française, ce qui est une omission importante, car le narrateur personnifie les montagnes. Au lieu de cela, la traductrice surinterprète en ajoutant «comme ils le peuvent».

- Aux lignes 11 et 12, le mot "éponger» est inadéquat du fait qu'on dit tout simplement "essuyer son front ", comme l'exprime prosaïquement la version russe. Ensuite, ce sont les montagnes qui essuient leur front avec les nuages et non «sur les nuées », "nuées» étant de plus d'un niveau de langage trop élevé comparé à l'original. Le mot « harassé » ne rend pas bien non plus nakhmourennye, ce terme russe qui est difficile à cerner, car il contient plusieurs émotions à la fois: être assombri, maussade, mécontent, triste, mélancolique, fâché, menaçant, préoccupé ou même concentré sur son travail ou bien plongé dans ses pensées. Sa racine est celle du mot «nuage», d'où l'image très visuelle de quelqu'un dont le front (lieu symbolique de la réflexion, de l'action intérieure) ressemble au ciel qui s'ennuage peu à peu, à l'opposé du ciel clair, calme et lumineux. Dans ce front expressif, quelque chose mijote, quelque chose se passe. L'équivalent employé en français, "harassé», n’est pas le mieux choisi car il évoque trop l'idée de fatigue. Encore une fois, la traductrice surinterprète, comme si elle tentait de préserver à tout prix la cohérence des idées (voir la référence à la lassitude aux lignes 7 et 8). Pourtant, ce mot, qui passe du langage naturel pour désigner une réalité abstraite, est important parce qu'il englobe en lui les deux univers, la nature et l'humain, et fait, du même coup, la synthèse du poème dans lequel la nature est personnifiée.

- Quant à la traduction des deux dernières lignes du poème, qui sont les plus intenses, elle est difficile à justifier. Nous y reviendrons. Restant encore sur le plan sémantique, nous remarquons que l'original «Marchent vers le haut et tout droit» n'équivaut pas à la version française "S'élèvent droites les cimes». Encore une fois, le dynamisme des montagnes personnifiées est réduit à une contemplation passive. Quant à la dernière ligne, au lieu de «Et traînent [tirent] la mer aux [vers les] cieux», Christiane Loré a 
opté pour «Tirant la mer jusqu'aux étoiles». La substitution des étoiles aux cieux provoque une déception chez le lecteur bilingue, d'autant plus qu'il s'agit du dernier mot qui porte en lui tout seul le poids du poème.

Les divergences qui surgissent à la suite de cette comparaison entre les contenus sémantiques de l'original et de sa traduction permettent d'éclairer les présupposés théoriques et par là l'interprétation du poème qui a pu guider la traductrice dans ses choix terminologiques, car la traduction n'est jamais un travail accompli à partir d'une subjectivité réduite à zéro. En herméneutique, on appelle ce moment la précompréhension dans laquelle se dessine l'horizon interprétatif qui mènera vers une compréhension. Pour ce faire, nous traiterons le poème traduit comme autonome et indépendant de l'original.

Il n'est pas rare de constater que l'horizon des traductions poétiques est souvent conditionné par l'hypertextualité, selon le terme de Berman (1973) ${ }^{7}$, qui motive et limite le travail tracé d'avance par la tradition poétique existante dans le pays d'accueil, les normes, les canons, les clichés, les expressions toutes prêtes. Dans cet horizon pénètrent aussi des modes de traduction et des courants théoriques et critiques qui prédominent dans un espace culturel. Cela rejoint de près la position de Meschonnic, qui critique l'effet structuraliste dans le domaine de la traduction poétique en France, position qui semble être confirmée dans le cas qui nous intéresse.

Nous tenterons donc de comprendre l'horizon interprétatif de la traductrice française de Chalamov.

À la suite de notre analyse du contenu sémantique, nous nous sommes rendu compte que la version française ne colle pas entièrement à l'original et que, de plus, ces changements de significations ne sont même pas motivés par le souci de sauvegarder la forme, qui semble au contraire être laissée de côté. La traduction ne reproduit ni le rythme monotone du russe (l'alternance de neuf et de huit syllabes) ni les quatrains aux rimes croisées ( $\mathrm{abab} / \mathrm{cdcd} / \mathrm{efef} / \mathrm{ghgh}$ ), où, à l'exception de la troisième strophe avec ses rimes complètes, les nonasyllabes $(\mathrm{a}, \mathrm{c}, \mathrm{g})$ se distinguent par la rime assonante $^{8}$ (concordance des sons or, oug, jam).

Avant tout, on note que, dans la traduction, le mouvement et le dynamisme, soulignés par les verbes d'action s'étirer, se profiler, marcher, traîner, sont atténués au profit de la description statique et méditative. D'où vient ce changement d'optique? L'original se lit au rythme d'une marche ou plus précisément au rythme d'une escalade: le sujet qui parle monte une montagne et observe la transformation du paysage qui se déploie devant ses yeux. Mais au lieu de parler de lui ou de ses propres impressions, il fait, en quelque sorte, parler et agir la nature. Sa marche commence en bas de la montagne, là où la route se trouve au niveau de la mer. Au fur et à mesure qu'il monte, il suit de son regard les montagnes qui se profilent et dont les crêtes s'approchent des nuages et du ciel. Durant cette escalade, la perspective de vision change brusquement: en bas, la mer n'est qu'une couche d'eau très mince alors que la montagne s'impose par sa masse gigantesque, mais au sommet, celle-ci disparait sous les pieds, laissant toute la place à la mer qui se confond à l'horizon avec le ciel. Le narrateur devient l'observateur de la nature qui, grâce à son regard, se met en mouvement et agit jusqu'à traîner la mer aux cieux. D'un autre côté, le fait qu'il regarde dehors lui permet de prendre conscience qu'il est lui-même en mouvement et que, sans sa présence, le monde extérieur ne serait qu'inertie apparente. Du même coup, les dimensions des montagnes et de la mer deviennent relatives selon le point d'observation. 
Quelle est maintenant l'interprétation proposée par la traductrice? L'analyse de la version française nous laisse croire que le narrateur contemple passivement le panorama, étant probablement déjà au sommet. Dans la première strophe, le russe dit que la route s'étire vers le haut et non au-dessus de la rivière. De même, le début de la deuxième strophe «Dans les nuits nordiques transparentes» devient «Sur la nuit transparente du Nord ». Et le troisième indice se trouve dans le dernier quatrain : au lieu des monts qui marchent, les cimes s'élèvent. Ce changement de perspective, si menu qu'il soit, n'est pas sans importance pour l'ensemble de l'interprétation du poème en français. On a l'impression que celui-ci recrée une image à trois niveaux: la route est au-dessus de la rivière, les monts, épuisés se cramponnent aux poteaux et leurs cimes s'élèvent droites. Et tout l'ensemble se renferme dans un cadre pictural sur le fond d'une nuit transparente. Le choix du singulier, dans le cas de la nuit (1. 5), prouve également que la traductrice considère l'ensemble du poème comme un bref instant contemplatif qui se passe ici et maintenant. Une autre confirmation nous fournit le dernier mot du poème «étoiles» alors qu'en russe nous lisons «cieux». Ce changement sémantique peut être justifié par le souci du rythme et de la sonorité. De ce point de vue, nebesa est mieux rendu par «étoiles» que par un mot court se terminant par une voyelle fermée "cieux». Mais ce changement valait-il vraiment la peine? Sauvegarder un aspect formel et détruire une image forte et éloquente se justifie-t-il?

Qu'est-ce que signifie ce choix dans l'interprétation du poème? Les étoiles évoquent visuellement la nuit, la noirceur, l'obscurité. Émotivement, elles font penser à l'angoisse, à la peur, à l'incertitude, aux chemins égarés. Mythologiquement parlant, nous sommes dans le domaine du chaos originaire, des forces maléfiques, du mal, de la perdition et de l'enfer. Dans l'ensemble du poème, toutefois, cette image peut être comprise comme la fin du spectacle: une fois le panorama contemplé, nous tirons les rideaux, nous éteignons les lumières et ce qui est vu retombe dans le vide de la noirceur jusqu'à la prochaine représentation.

Les changements sémantiques introduits avec une telle aisance par la traductrice sont probablement motivés par l'approche structuraliste qui vise avant tout l'ensemble formel du poème, les jeux de signes et leurs relations mutuelles dans un système cohérent. Les signifiants «cieux» et «étoiles », par exemple, appartiennent à la même famille. Dans cette logique, ils sont interchangeables, d'autant plus que les «étoiles» seront en parfaite cohérence avec la «nuit» (au singulier en français!) qui est évoquée dans la deuxième strophe. De même, l'équivalent «harassé » rejoint facilement l'idée des montagnes épuisées sous le poids de la rivière.

Au-delà de la distinction forme/contenu, ce qui prime est le poème lui-même. Réfléchir seulement à partir de la forme réduit le poème à l'esthétisme ou à la virtuosité langagière dont seul le plus grand poète est capable et devant laquelle tout traducteur se sent désarmé et impuissant. Il est vrai que l'union heureuse qui existe dans la poésie entre la langue et les significations que celle-ci véhicule est un fait indéniable: c'est sa force redoublée. C'est la nature véritable de la poésie et même la position opposée qui vise à briser cette union ne fait que confirmer la règle. Mais il est vrai aussi que chaque poème dit quelque chose ou au moins fait entendre quelque chose à celui qui est prêt à lui tendre l'oreille. Le poème pose des questions, cherche des réponses, provoque des réactions d'ordre émotif, déstabilise des terrains connus, mobilise l'attention du lecteur parfois jusqu'au paroxysme. Si l'on traduit la forme 
sans accorder trop d'importance à la force qui émane du poème, celui-ci survit comme mutilé s'il n'est pas anéanti d'avance.

Comment surpasser l'imposture du signe, pour reprendre l'expression de Meschonnic, et sa dualité? De quelle manière rendre la même force d'expression dans une autre langue? Nous proposons l'analyse dialogique comme terrain de réflexion sur ce point. Inspirée de la pensée bakhtinienne, cette analyse, notons-le, n'est pas une stricte application d'une théorie toute prête: nos propos ne pourraient pas être justifiés dans le cas qui nous intéresse, car Bakhtine considérait que la poésie était monologique, contrairement à la prose, au roman en particulier.

Nous pensons cependant que la poésie, tout comme n'importe quel acte de création artistique ou langagière, prend ses origines dans l'heteroglossia, dans la pluridiscursivité, dans la multiplicité de voix, même s'il est vrai aussi qu'en poésie c'est souvent la forme qui s'impose aux idées et trace son chemin au contenu. «La poésie, c'est freiner le flux sonore, et couler la lave des sons dans les formes du sens », comme l'explique Varlam Chalamov (1993: «De la nature du vers russe», 126). Néanmoins, un poème se comprend dans ses réseaux discursifs initiaux, c'est-à-dire à partir du questionnement qui précède la création artistique et qui mène à poser le geste de l'écriture. L'ensemble de l'œuvre d'un auteur peut s'avérer éclairant ainsi que les relations qu'il entretient avec d'autres voix ou présences réelles ou virtuelles incluses dans le dialogue, qu'elles soient proches ou lointaines du point de vue spatio-temporel. La poésie s'adresse à l'autre, elle lui pose des questions, elle entend ses réponses qui soulèvent, à leur tour, de nouvelles questions ${ }^{9}$. Le poème ne naît jamais à partir de la certitude, sinon il ne serait pas poème.

Pour comprendre le dialogue initial de «La chaussée», il suffit de puiser dans la prose, les récits autobiographiques et la poésie de Varlam Chalamov. À propos du sens de la création poétique, il dit: «L'âme de la poésie, c'est l'actualité. C'est aussi en cela que consiste la citoyenneté: se faire l'écho de tous les événements de son pays et de son époque [...]»(1993: «De la poésie au camp», 93). Mais c'est aussi la communication avec Dieu (1993: «De la poésie au camp», 86).

Le poète, appelé par ses premiers critiques «le chantre du Grand Nord» (Chalamov 1993: "Le Grand Nord dans mon œuvre», 21), explique également ce que signifie pour lui la nature omniprésente dans son œuvre: «Le sentiment de la nature, c'est la faculté de déceler dans les manifestations de la nature les mouvements de l'âme humaine, la faculté de prévoir et de prédire le cours des événements» (1993: «De la poésie de la nature», 95). Et plus loin: «En explorant la nature à travers le vers, le poète y trouve des correspondances avec ses propres angoisses et son propre état d'âme» (1993: «De la poésie de la nature», 96-97). Quant au lecteur, il «cherche toujours une réponse aux problèmes «éternels» [...]. Il veut une solution aux problèmes vitaux, une réponse sur le sens de la vie, sur les liens de la vie et de l'art» (1993: «De la prose», 27).

Grâce aux écrits du poète lui-même, nous sommes lancés sur des pistes déjà bien tracées. Dans l'événement d'un poème se croisent inévitablement la vie concrète du poète, le monde familier qui l'entoure, la présence de Dieu, le rôle de l'art et le dialogue avec le lecteur éventuel. Dans la poésie de Chalamov se dessinent les images chères au poète: la mer, le ciel, les nuages, la glace, l'arbre, la barque, le chemin. Nous nous limiterons, cependant, à l'image de la mer liée au ciel, car elle contribue d'une manière importante au sens du poème «La chaussée». 
Dans «Le pin nain ${ }^{10} »$, voici comment le poète définit le ciel:

Il [le pin nain] tend vers le ciel, là-bas

Où n'est ni peine ni tourment

$\mathrm{Ni}$ crocs de glace semeurs de mort

Ses mains décharnées, noires et sales. (p. 139)

Ce lieu de paix est pourtant loin, comme nous l'apprend un autre vers intitulé Toast en l'honneur de l'Aian-Uriah:

Je porte un toast $[\ldots]$

À la ration de pain gluant Engloutie en toute hâte Au ciel pâlot, et trop haut,

À la rivière Aian-Uriah! (p. 173)

Mais ce ciel, qui est trop haut, semble indispensable à la vie. Parfois, il ne suffit que de lui tendre la main:

Je ne vis pas seulement de pain

Dans le froid noir du petit matin

J'ai trempé dans la rivière

Un morceau de ciel clair... (p. 145, sans titre)

Ici, l'élément de l'eau (fluidité insaisissable en mouvement) se rencontre avec le ciel. Dans Nuit de lune, seule la nuit détruit cette heureuse liaison:

Qui dira, sous la lune,

Où est l'eau, où est le ciel,

Où est jetée la senne,

Où l'on tire les filets. (p. 161)

À ce tableau déjà éloquent, il ne reste que de joindre la définition de la vie:

$[\ldots]$

Et la vie, fugitive, se mesurait

À l'aune de principes terrestres

Qui sur la planète président à nos destins,

Maîtres de vie ou de mort

Juste faits pour demeurer

Un temps, en secret.

Ainsi, sauvant nos âmes,

Ils guident entre les écueils

Ceux-là seuls qui leur sont soumis

Et n'ont pas recherché la vie... (p. 141, sans titre)

Bien que fugitive, la vie, avec ses principes terrestres, possède ses lois parfois imperceptibles aux humains mais qui semblent être justes, selon le poète. Ceux qui savent vivre se laissent porter par le courant de l'eau et guider par le destin, comme l'a fait Noé (p. 155, sans titre). Ceux qui «n'ont pas recherché la vie» sont peut-être ceux qui savent tremper une mie de pain dans le ciel ou pêcher une étoile dans l'eau lors d'une nuit de lune ou encore aller au-delà de ses propres limites.

Le dialogue que mène Chalamov dans ses écrits sert à éclaircir le sens de la dernière ligne du poème «La chaussée». Les deux images "tirer la mer vers les cieux» 
(original) et «tirer la mer jusqu'aux étoiles» (version française) suggèrent deux interprétations différentes du poème.

Selon nous, cette dernière ligne du poème constitue une synthèse non seulement de son contenu (animé/inanimé, stabilité/mouvement, vie/mort, horizontalité/verticalité) mais aussi de la vie et de l'art, tels que vus par le poète. La rencontre de la mer avec le ciel est très évocatrice. Imaginons le bleu de la mer et le bleu du ciel, séparés par une ligne fine de l'horizon. L'une, dynamique avec ses vagues qui s'élèvent vers le haut, mais qui retombent aussitôt soumises à la gravitation, évoque la condition humaine. L'autre, par contre, statique et paisible, affirme plutôt l'aspiration des humains à un au-delà qui serait plus juste et plus serein et qu'on peut peut-être atteindre à travers la création artistique. Ce bleu divisé en deux n'est qu'un seul bleu qui fait partie de chaque humain. De plus, le pluriel «les cieux» fait contraste avec la finitude de la vie terrestre et l'infini de l'univers où, derrière le ciel visible à l'œil nu, existent encore d'autres cieux imperceptibles et inexplorés.

Le tableau statique présenté dans la traduction française de «La chaussée» ne rend pas bien le dynamisme qui émane de l'original. La palette de couleurs choisie par la traductrice préférant noyer le bleu dans la noirceur détruit l'impact du poème que l'on ressent à la lecture de l'original. Les «étoiles» font du ciel un simple décor. D'où la conclusion que certains mots ne peuvent pas être rendus par n'importe quel terme appartenant à la même famille de signes. Christiane Loré semble rester fidèle à la pensée structuraliste selon laquelle un signe renvoie toujours à un autre signe et non pas à la réalité elle-même ${ }^{11}$. Ainsi, elle se laisse guider par le principe d'interchangeabilité de mots. À un autre endroit, elle remplace «les nuages» par «le ciel», comme si le ciel, les étoiles et les nuages signifiaient exactement la même chose. Voici le poème "Camée», que nous citons entièrement:

Au déclin de l'âge, sur la pente des monts

J'ai taillé ton portrait dans le roc.

Plus sûrs que la plume gracile,

La hache, le pic et la cognée.

Au pays du gel et des mâles

Et des visages tôt burinés,

Sans espoir, simple vanité,

Les traits d'une femme j'ai évoqués.

Lors, dans l'anneau de neige

J'ai serti ton profil de pierre.

Puis redoutant l'obsédant regret

J'ai celé l'anneau dans le ciel. (p. 147)

La dernière ligne qui se lit en russe «J'ai caché l'anneau dans les nuages» met l'accent sur la matérialité des nuages qui remplacent un papier en soie ou un morceau de velours dans lesquels on emballerait des objets précieux. Le nuage est aussi plus près du narrateur que l'est l'immensité du ciel, comme si le camée restait à portée de la main au lieu de disparaître quelque part dans l'espace.

Le dialogue, quelle que soit la forme sous laquelle il se présente, n'est jamais une fusion confuse, un tourbillon de voix amalgamées. Il n'est ni synthèse ni répétition car, tout imbibé d'émotions, il inclut au moins deux interlocuteurs qui se parlent, qui 
se posent réellement des questions auxquelles les réponses doivent être données ne serait-ce que sous forme de questionnement. À qui s'adresse Chalamov? Et quelles questions pose-t-il? Chalamov, comme tant d'autres de ses contemporains, était l'un de ceux que Grigori Svirski appelle «écrivains de la liberté» dont l'art est celui de la résistance (Svirski 1981). Il est évident que Chalamov s'adresse à la fois aux autres poètes de son époque qui partageaient le même destin tragique ainsi qu'à leurs bourreaux communs. La réflexion sur le sens de l'art n'est au fond qu'une question posée sur le sens de l'existence et de la souffrance du poète condamné au bagne. Dans «La chaussée », Chalamov semble justifier son choix et affirmer sans cesse l'importance de l'art dans cette période de noirceur et de destruction. Quand Boris Pasternak, son destinataire épistolaire, remet en question sa propre création artistique, Chalamov lui répond ainsi: "[vos] poèmes avaient une vie, une force grâce auxquelles, je le répète, des gens sont restés des êtres humains» (Chalamov 1991: 43). C'est son oui à la tradition et à la continuité de la culture qui a vu son jour dans le bassin méditerranéen, position également soutenue par Mandelstam, Pasternak, Zamiatine et tant d'autres. L'art sert également d'opposition à l'avilissement de l'homme: «L'homme russe, âme humaine, âme vivante, est une victime de la politique. Depuis des siècles, la politique piétine cette âme vivante avec des bottes des soldats et des gardes-chiourmes» (Chalamov 1991: 139). L'image poétique de la mer tirée vers les cieux, dans le poème de Chalamov, rejoint, sans aucun doute, la même position esthétique.

Et le poète? À maintes reprises, Chalamov appuie le lien inébranlable qui existe entre la poésie et la vie, et rejette, par conséquent, le pur esthétisme et la séduction artistique $^{12}$. À ce propos, Czeslaw Milosz évoque les mémoires d'Alexander Wat (poète polonais et victime du stalinisme) et de Nadejda Mandelstam (veuve du poète russe persécuté) où l'on retrouve la même conclusion: «le poète n'a pas le droit de devenir un séducteur, c'est-à-dire d'utiliser ses dons pour faire de son lecteur un adepte de quelque idéologie inhumaine» (Milosz 1983: 123). Dans son poème autobiographique "Cher levain du monde», Mandelstam, par exemple, s'identifie à un mendiant qui retrouve sur son chemin des traces oubliées et les suit comme un guide aveugle et étranger à lui-même. Dans un autre poème intitulé "Mon siècle», le narrateur assume le rôle symbolique de l'agneau sacrifié s'offrant comme une source de nourriture inépuisable. Le poète parle au nom de toute une génération d'artistes sacrifiés qui se retournaient vers le passé pour en puiser les bribes de sens au milieu du chaos et du mensonge. Plus tard, Iossip Brodski, un autre poète accusé, aura le courage de défier l'autorité totalitaire en affirmant lors de l'interrogatoire mené par la juge Savaleva que sa poésie venait de Dieu ${ }^{13}$.

Lire ou traduire un poème de Chalamov, tout en préservant la force de sa parole, devient possible quand on est à l'écoute de la communication, sinon de la communion, qui existe entre les compagnons de misère. En dehors de ces liens de solidarité cimentés par le dialogue, le poème "La chaussée» n'est au premier niveau qu'une contemplation de la nature ou, au sens plus profond, une réflexion sur l'inspiration artistique et non une défense de l'art au service de la vie, une défense de l'existence humaine individuelle dans sa dimension créatrice et spirituelle.

La voix sobre et ferme du dissident Chalamov retentit pour témoigner de son époque, de son vécu, de ses choix en tant que citoyen et poète. Ce témoignage se fait également au nom des autres ou même à la demande des autres, comme le décrit Akhmatova dans son Requiem (1966: 14-15): 
Dans les années terribles de la «Iéjovchtchina ", j'ai passé dix-sept mois à faire la queue devant les prisons de Léningrad. Un jour, quelqu'un a cru m’y reconnaître. Alors, une femme aux lèvres bleuâtres qui était derrière moi et à qui mon nom ne disait rien, sortit de cette torpeur qui nous était coutumière et me demanda à l'oreille (là-bas, on ne parlait qu'en chuchotant) :

— Et cela, pourriez-vous le décrire?

Et je répondis:

- Oui, je le peux.

Alors, une espèce de sourire glissa sur ce qui avait été jadis son visage.

Le bleu qui englobe la mer et le ciel, c'est l'homme et l'artiste, la vie et l'inspiration, la nature et la culture, ici et ailleurs, les deux côtés d'une seule réalité si farouchement défendue par les artistes qui incarneront pour toujours ce que Dostoïevski a appelé la «beauté crucifiée».

\section{CONCLUSION}

La traduction dialogique est celle qui prend en considération la voix qui parle, son intonation et ses interlocuteurs. La forme n'est qu'un moyen qui s'actualise et par lequel la voix s'exprime pour rejoindre l'autre. On analyse la forme mais on écoute la voix. L'une se prête à l'observation et l'autre se dérobe, insaisissable, présente et absente à la fois. Le traducteur reste neutre, à l'extérieur de la forme, distant à son égard au moment où il procède à l'analyse de ses divers principes et niveaux d'organisation. Quant à la voix, elle invite, convoque et engage au dialogue. Elle déstabilise, provoque et responsabilise ceux qui l'écoutent. La traduction, dans ce cas, n'est pas uniquement un exercice esthétique mais un échange mutuel enrichissant dans lequel l'entente ou le désaccord, l'affirmation ou la négation, la confirmation et l'interrogation se côtoient continuellement. On a le droit de dire «oui» ou «non» à Chalamov, mais il est primordial de se mettre tout d'abord à l'écoute de sa parole. Car la visée, du point de vue dialogique, n'est pas la poésie qui transcende la lecture individuelle et suit une interprétation établie par la culture, la tradition, l'époque ou le courant de pensée, mais celle qui vise à rejoindre la voix de l'autre dans toutes ses nuances et sa richesse d'expression.

«L'une des particularités de la poésie, écrit Chalamov, — une de ses qualités est que par sa nature, par son authenticité, elle s'adresse non à l'humanité, mais à l'individu, elle lui parle "les yeux dans les yeux".» (1991: 55)

\section{NOTES}

1. Nous employons ce terme au sens que lui est donné par François Laplantine et Alexis Nouss (1997: 85): «[...] le métissage n'existe pas seulement dans la non-coïncidence, mais dans la non-résolution. Il ne saurait être de l'ordre de la symbiose, ni de la synthèse, c'est-à-dire de l'accomplissement. Pas plus en avant qu'en arrière, dans le point de départ que dans le point d'arrivée [...], il est le devenir plus qu'avenir et appelle à être pensé en lui-même dans son inachèvement. Transitoire, imparfait, inachevé, insatisfait, le métissage est toujours dans l'aventure d'une migration, dans les transformations d'une activité de tissage et de tressage qui ne peut s'arrêter. C'est dire combien cette notion est éminemment contradictoire. Elle ne peut être mobilisée comme une réponse, car elle est la question elle-même qui perturbe l'individu, la culture, la langue, la société dans leur tendance à la stabilisation.»

2. Foucault explique que le signe binaire apparaît à l'âge classique, à l'avènement d'une nouvelle rationnalité qui brise l'union primaire et directe basée sur la ressemblance naturelle entre les mots 
et les choses, qui dominait l'épistémè de la Renaissance, en y introduisant la représentation comme lien entre le signe et la chose désignée (1966: 58). Dès lors, l'ordre des choses devient historique et le langage arbitraire (1966: 77). De même, l'organisation du savoir se fait par le biais des actes de connaissance et non plus par la lecture ou l'interprétation du monde vu comme un grand livre ouvert qui se donne à lire $(1966: 32)$.

3. Les visées critiques de Berman et d'Etkind rejoignent le propos de Meschonnic. Antoine Berman (1995: 29) constate que les traductions françaises font voir les poètes comme des «libertins» au lieu de les faire découvrir dans leur plénitude (chrétien, prosateur, etc.). À la page 47, il définit ainsi «la psychè traductive»: «[...] un certain laisser-aller, une certaine désinvolture allant jusqu'au mépris objectif de l'auteur (et du public), une complaisance narcissique et, last but not least, un manque de consistance [...] ». D’un autre côté, Efim Etkind (1982: 13) pense que «[1]e mal dont souffre depuis longtemps la traduction poétique française porte un nom: c'est la rationalisation systématique de l'original, qui ignore l'unicité irréductible de chaque poème».

Il existe peut-être une autre hypothèse: la culture littéraire française du XIX ${ }^{e}$ siècle, tout comme la culture anglaise, est avant tout romanesque et non poétique, comme l'est, par exemple, la culture polonaise de la même époque. Les grands prosateurs qui dominent l'espace littéraire du siècle passé perfectionnent donc la langue du roman; celle de la poésie, outre les recherches avant-gardistes, restent probablement plus canonisée et rattachée au passé. Cette hypothèse est inspirée de Maria Janion (1991: 128) : «Conrad et le dilemme du romantisme polonais».

4. La problématisation du discours esthétique est traité par Terry Eagleton (1990). Dans une interview accordée à Maryse Souchard ("On the Concept of the Aesthetic», dans Recherche sémiotique Semiotic Inquiry, vol. 10, $\mathrm{n}^{\text {os }} 1-3,1990$, Montréal, Association canadienne de sémiotique), Terry Eagleton explique: «As Adorno recognized, there is no immediated immediacy, and it has been one of the illusions of the bourgeois aesthetic tradition to think that there was an immediacy which was not somehow mediated. We must cling to a suspicion of that immediacy.» (p. 173)

5. Cette tension qui existe entre le langage littéraire contemporain et la normativité d'un langage officiel appartenant à une longue tradition est un véritable problème de traduction. L'article de Maryla Laurent, "L'écriture "impressionniste" de Tadeusz Konwicki dans un français "cartésien" (1997: 53-67), en est particulièrement évocateur. Elle insiste sur le fait que l'impressionnisme langagier de Konwicki, son style particulier, qui exprime à travers le langage le conflit que le narrateur entretient avec la réalité, ne passe pas en français, qui exige de ses usagers le respect de règles bien définies. Quelques-uns de ses commentaires illustrent la position conflictuelle qu'occupe la traductrice déchirée entre les libertés de l'auteur polonais et la rigidité de la langue française:

«Parfois la répétition est tout simplement exaspérante [...]» (1997: 63).

«Le polonais semble avoir des tolérances que la langue de Molière ignore dans la mesure où il se réfère plus volontiers à l'intonation de la langue parlée» (1997: 63).

«Le pléonasme est particulièrement persécuté en français» (1997: 64).

«La contamination d'un niveau de langue par un autre est d'un effet particulièrement malheureux» (il s'agit du trait stylistique propre à Konwicki) (1997: 65).

"La syntaxe polonaise a ici une structure déficiente [...].» La traductrice se demande s'il s'agit d'une transgression volontaire de la part de Konwicki ou bien d' "une maladresse malencontreuse » (1997: 66).

Au moment où le narrateur hésite quant à l'identité sexuelle d'un personnage, la traductrice surinterprète en disant: «Il était impossible de faire autrement (une dame qui semble de sexe féminin est un non-sens) et, par ailleurs, il nous semblait déceler là une faiblesse de la technique narrative qui nous était insupportable chez un grand auteur.» (1997: 66).

Bakhtine (1979: 347) a saisi le même problème dans son étude de Dostoïevski: «La conscience scientifique de l'homme moderne lui a appris à s'orienter au milieu de la complexité de «l'univers des probabilités» sans se troubler des «indéterminations»; il sait même les faire entrer dans ses calculs. Cette conscience s'est déjà familiarisée avec l'univers d'Einstein, avec la pluralité des systèmes de référence, etc. Mais dans le domaine de la connaissance artistique, on continue souvent d'exiger une détermination grossière et niaise qui, de toute évidence, ne peut plus convenir.»

6. Depuis Platon, l'accès à la connaissance se fait à l'aide de la vision.

7. À ce sujet, voir aussi Berman (1985: 31-150).

8. La rime assonante de Chalamov était sévèrement critiquée par Boris Pasternak qui la définissait comme « $[\ldots]$ les rimes fausses, incomplètes, qui mènent à une langue négligente et au manque d'équilibre, à l'état de flou de l'ensemble [...]» (Chalamov 1991: 33), à quoi répliquait Chalamov: «Là, il me semble que vous avez tort, car la rime n'est pas seulement l'armature et la serrure du vers, 
elle n'est pas seulement la pièce maîtresse, la clé de l'harmonie: elle est - et c'est sa principale raison d'être - un instrument servant à la recherche des comparaisons, des métaphores, des pensées, des inversions, des images, un aimant puissant pointé vers les ténèbres et survolé par l'univers entier, ne déposant dans le poème qu'une infime partie de ce qu'il a attiré. La rime est l'instrument du choix, l'outil de la pensée poétique et de la connaissance du monde [...]» (Chalamov 1991: 43).

9. De tout autre avis est Eugenio Coseriu (1997: 91) qui dit: «Siamo persuasi [...] che la poesia non sia indirizzata ad un altro, che quel che importa al poeta è sempre solo la obiettivazione di se stesso. [...] [L]a poesia è l'attività di un soggetto universale, il poeta assume la soggettività universale nell'instante in cui sta poetando. Allora non è un parlante fra gli altri, ma realizza il parlare in modo assoluto $[\ldots]$ ». Voici notre traduction: «Nous sommes persuadés [...] que la poésie ne s'adresse pas à l'autre, que ce qui compte pour le poète est toujours et seulement l'objectivation de soi-même. [...] [L]a poésie est une activité d'un sujet universel et le poète assume la subjectivité universelle au moment de la création poétique. Il n'est donc pas un sujet parlant parmi les autres mais il réalise le parler dans un mode absolu.»

10. Les poèmes de Chalamov qui sont inclus dans cette partie de notre analyse sont publiés dans le même volume Tout ou rien (1993). Les pages de références sont indiquées directement dans le texte.

11. «Le sens des mots [...] est décidément un fait linguistique — disons, pour être plus précis et moins étroits, un fait sémiotique. Contre tous ceux qui assignent le sens (le signifié) non au signe, mais à la chose elle-même, le meilleur argument, et le plus simple, serait de dire que personne n'a jamais goûté ni humé le sens de fromage ou de pomme. Il n’y pas de signifié sans signe.» (Jakobson 1963: 78-79)

12. «L'objet de la poésie est le perfectionnement moral de l'homme [...]» (Chalamov 1991: 54).

13. Voici le fragment de l'interrogatoire:

«- Quelle est votre profession?

— Je suis poète. Je suppose...

— Pas de ces «je suppose» ici. Tiens-toi droit. Ne t'appuie pas contre le mur. Regarde le tribunal.

As-tu une profession stable?

- Je croyais que c'était là une profession stable.

— Mais quelle est ta spécialité, d'une manière générale?

— Je suis poète, traducteur poète.

- Et qui t'a reconnu comme poète? Qui t'a fait entrer dans les rangs des poètes?

— Personne. Et qui m'a fait entrer dans les rangs de l'espèce humaine?

- As-tu étudié pour l'être?

- Quoi?

— Pour être poète. N'as-tu pas cherché à poursuivre tes études au lycée, où l'on prépare, où l'on apprend?

— Je n'ai pas cru que c'était matière d'enseignement.

- Comment alors?

— Je crois que ça... vient de Dieu...»

Cité par Pierre Emmanuel (1966: 11-12).

\section{ANNEXE}

Chalamov, Varlam (1991): Correspondance avec Boris Pasternak et Souvenirs, tr. Sophie Benech et Lily Denis, Paris, Gallimard.

Pasternak à propos de la poésie et de la prose:

«Tout ne peut être dit en vers. On comprend et on ressent les poèmes de Lermontov avec plus de finesse et de profondeur si l'on garde sa prose à l'esprit. La prose est un matériau qui permet une meilleure compréhension de la poésie. Prenez Verlaine, qui n'a rien écrit en prose: on a besoin de la peinture française de son époque pour le saisir pleinement.» (p. 191)

Pasternak:

«Je n’apprécie guère l'ironie romantique. La poésie, comme la vie, est chose trop sérieuse, il n'y a pas là matière à plaisanter. On ne s'en sort pas par une boutade. L'ironie est une mauvaise arme, un mauvais bouclier...» (p. 192) 


\section{RÉFÉRENCES}

Акнmatova, Anna (1966): «En guise de préface», Requiem, tr. Paul Valet, Paris, Éditions de Minuit.

Bakhtine, Mikhaïl (1979): La poétique de Dostoüevski, tr. Isabelle Kolitcheff, Paris, Seuil.

Berman, Antoine (1973): "Traduction ethnocentrique et hypertextuelle», L'écrit du temps. La décision de traduire: l'exemple de Freud, Paris, Éditions de Minuit.

_ (1985): «La traduction et la lettre ou l'auberge du lointain», Les Tours de Babel. Essais sur la traduction, Mauvezin, Trans-Europ-Repress.

- (1995): Pour une critique des traductions: John Donne, Paris, Gallimard.

Blanchot, Maurice (1955) : «L'expérience originelle», dans L'espace littéraire, Paris, Gallimard. Buber, Martin (1969): Je et tu, tr. G. Bianquis, Paris, Aubier.

Chalamov, Varlam (1991): Correspondance avec Boris Pasternak et souvenirs, tr. Sophie Benech et Lily Denis, Paris, Gallimard.

- (1993): Tout ou rien, tr. Christiane Loré, Lagrasse, Verdier.

Coseriu, Eugenio (1997): Linguistica del testo. Introduzione a una ermeneutica del senso, Rome, La Nuova Italia Scientifica.

Eagleton, Terry (1990): The Ideology of the Aesthetic, Oxford et Cambridge, Basil Blackwell.

Emmanuel, Pierre (1966): «Un poète métaphysique en U.R.S.S.», Collines et autres poèmes, Paris, Seuil.

ETKInd, Efim (1982): Un art en crise. Essai de poétique de la traduction poétique, Lausanne, L’Âge d'Homme.

Foucault, Michel (1966): Les mots et les choses. Une archéologie des sciences humaines, Paris, Gallimard.

Jаковson, Roman (1963): "Aspects linguistiques de la traduction», Essais de linguistique générale, Paris, Éditions de Minuit.

Janion, Maria (1991): Projet de critique fantasmatique, Varsovie, PEN.

Laplantine, François et Alexis Nouss (1997): Le métissage, Paris, Flammarion.

Laurent, Maryla (1997): «L'écriture «impressionniste» de Tadeusz Konwicki dans un français "cartésien" ", Traduction comme moyen de communication interculturelle (Elzbieta Skibinska, dir.), Acta Universitatis Wratislaviensis, $\mathrm{n}^{\circ}$ 1990, Romanica Wratislaviensia XLIV, Wroclaw, Wydawnictwo Uniwersytetu Wroclawskiego, 1997, p. 53-67.

Lévinas, Emmanuel (1974): Totalité et infini. Essai sur l'extériorité, 4e éd., La Haye, Martinus Nijhoff.

Meschonnic, Henri (1989): La rime et la vie, Lagrasse, Verdier.

Milosz, Czeslaw (1983): Témoignage de la poésie, Paris, PUF.

Petrosino, Silvano et Jacques Rolland (1984): La vérité nomade. Introduction à Emmanuel Lévinas, Paris, La Découverte.

Svirski, Grigori (1981): "Écrivains de la liberté. La résistance littéraire en Union Soviétique depuis la guerre», tr. Daria Olivier, L'art de la résistance, Paris, Gallimard. 